\title{
DS-CDMA with $M$-ary Orthogonal Modulation for Wireless Sensor Networks Simultaneously Monitoring Multiple Events
}

\author{
Ee How Sim and Lie-Liang Yang \\ School of Electronics and Computer Science \\ University of Southampton \\ SO17 1BJ, United Kingdom \\ Tel: 0044-(0)23-8059 3364, Email: 1ly@ecs.soton.ac.uk
}

\begin{abstract}
In this paper, we propose a novel WSN framework for one fusion center to monitor simultaneously multiple source events (SEs), each of which has multiple states. In the proposed WSN, every SE is observed by a range of local sensors, which convey their observations to the fusion center in the principles of direct-sequence code-division multiple-access (DS-CDMA) associated with $M$-ary orthogonal modulation (MOM). Therefore, the proposed WSN is referred to as the MOM DS-CDMA WSN. In the MOM DS-CDMA WSN, the local sensors monitoring the same $\mathrm{SE}$ transmit their signals to the fusion center in the principles of time-division multiple-access (TDMA), while the local sensors serving different SEs communicate with the fusion center in the principles of DS-CDMA. By making use of the advantages of MOM, the fusion center detects the SEs' states either coherently based on the maximal ratio combing (MRC) fusion rule, or noncoherently based on the equal gain combining (EGC) fusion rule. The detection performance of the fusion center is investigated by simulations. Specifically, the impacts of the number of SEs, the number of local sensors per SE, the observation reliability of local sensors, the reliability of the channels from local sensors to fusion center, etc., on the fusion detection performance are addressed.
\end{abstract}

\section{INTRODUCTION}

In WSNs, signaling and detection are the very important issues, which have received a lot of research in recent years [110]. Specifically, for two-state (or binary) events monitored, optimum and sub-optimum fusion detection algorithms have been proposed under various assumptions. Fusion detection rules found in literature include Neyman-Pearson detection [2, 9], Bayes detection [2, 5, 9], maximum likelihood detection [2$6,9]$, maximal ratio combining (MRC) detection and equal gain combining (EGC) detection [3-6], Chair-Varshney fusion detection [4-6], etc. In order to improve the spectral-efficiency and to reduce the detection delay, in [11], a multiple-access model has been proposed for transmitting signals from local sensors to fusion center and, furthermore, corresponding fusion detection rules have been proposed and studied. Additionally, in [7], the fusion detection of $M$-ary events has been investigated by merging the fusion detection with channel decoding. Owing to its low-complexity, in WSNs, noncoherent fusion rules are often preferred to the coherent fusion rules, which require extra complexity and extra resources for channel estimation [12].

In this paper, we propose and investigate a novel WSN framework, where multiple source events (SEs) are simultaneously monitored by a number of local sensors. We assume that each SE has $M$ number of states, determined by the states of one to several parameters. The proposed WSN has a parallel triplelayer network structure [4], where the SEs are monitored by the local sensors, which convey their observations to the fusion center over wireless channels. To be more specific, in the proposed WSN, after the observation of a SE, a local sensor transmits its decision (one of the $M$ states) to the fusion center using MOM modulation with the aid of DS-CDMA [13]. Hence, for brevity, we refer to it as the MOM DS-CDMA WSN. In the MOM DSCDMA WSN, the local sensors serving the same SE convey their decisions to the fusion center in the TDMA principles. Hence, these sensors do not interfere with each other. By contrast, the sensors serving different SEs transmit signals to the fusion center in DS-CDMA principles. In this case, the signals arriving at the fusion center simultaneously may interfere with each other, if they are not orthogonal due to the spreading codes used by the sensors and also the impairment introduced by wireless channels. In this contribution, two fusion decision rules are investigated associated with the MOM DS-CDMA WSNs, one is the coherent MRC fusion rule and the other one is the noncoherent EGC fusion rule. Since the MRC fusion rule requires the knowledge of all the channels from the local sensors to the fusion center, while the EGC fusion rule does not, the MRC fusion rule may demand much higher complexity than the EGC fusion rule. In this paper, the error performance of the MOM DS-CDMA WSN is investigated, when the channels from local sensors to fusion center experience independent Rayleigh fading. Our focus is on the impact of the number of SEs, the number of sensors per SE, the observation reliability of local sensors, the reliability of wireless channels, etc., on the detection performance of the MOM DS-CDMA WSNs.

From our studies and performance results, we observe that the MOM DS-CDMA constitutes one of the promising signaling schemes in WSNs for efficient transmission of signals from local sensors to fusion center. It is flexible to support multiple SEs, is suitable for operation with $M$-ary SEs and signals can be detected using either relatively higher complexity but better performance coherent fusion rules or low-complexity noncoherent fusion rules. The MOM DS-CDMA WSNs are capable of achieving promising detection performance at reasonable SNR level. Furthermore, our performance results show that, by deploying more local sensors, the EGC fusion rule is capable of attaining the same detection performance as the MRC fusion rule. In other words, the same detection performance of the MOM DS-CDMA WSN may be achieved either by the higher complexity MRC fusion rule using a lower number of local sensors or by the lower complexity EGC fusion rule with a higher number of local sensors. 


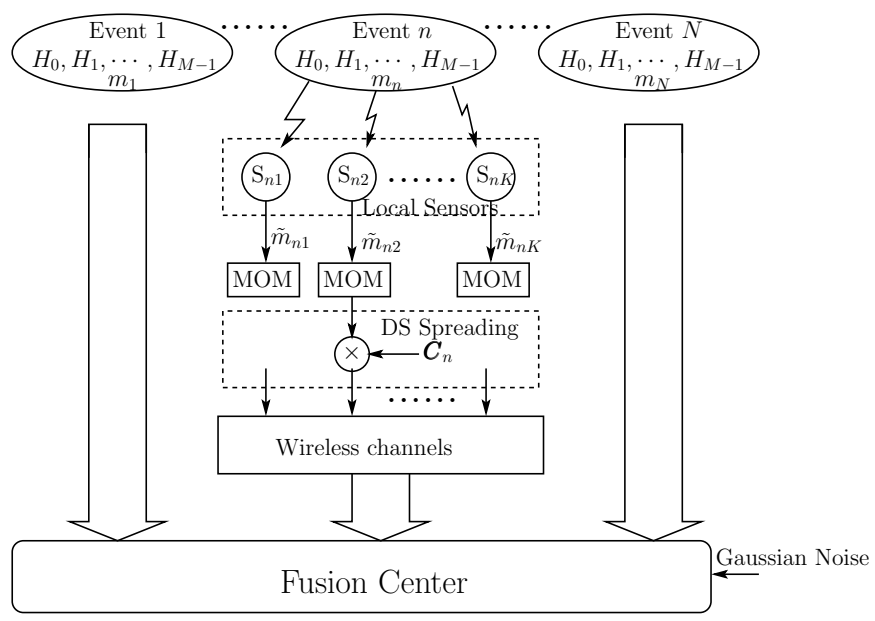

Fig. 1. Triple-layer system model for the MOM-DS-CDMA WSN monitoring multiple source events.

\section{System Description}

The sensor network considered in this paper is a typical triple-layer WSN model [4-10], which is shown as Fig. 1. There are $N \geq 1 \mathrm{SEs}$, each of which is monitored by $K \geq 1$ local sensors. Each SE has multiple parameters being monitored, which are represented by multiple states. After a local sensor observes a state of its SE, it conveys this state to the fusion center based on the MOM DS-CDMA principles, which will be detailed in our forthcoming discourse. Finally, at the fusion center, the states of the $N$ SEs are estimated based on the signals received from all the $K N$ local sensors. Below we describe in detail the operations carried out at the local sensors and at the fusion center.

\section{A. Sensor Processing}

In order to make the problem manageable, we assume that the $N$ SEs being observed are uncorrelated. Each of the $N$ number of SEs is monitored by $K$ local sensors, which observe the SE independently without interference with each other. We assume that the SEs have $M$ states, represented by $H_{0}, H_{1}, \cdots, H_{M-1}$. When the $n$th SE is at the state $H_{m_{n}}$, its local sensor, say $S_{n k}$, generates an integer value $\tilde{m}_{n k}=m_{n}$, if its observation is correct; Otherwise, it generates an integer value $\tilde{m}_{n k} \neq m_{n}$, when the observation is incorrect. Note that, $\tilde{m}_{n k}, m_{n} \in$ $\{0,1, \ldots, M-1\}$. We assume that all the local sensors have the same correct observation probability $P_{c}$. Correspondingly, the incorrect observation probability is $P_{e}=1-P_{c}$. Once a local sensor makes an erroneous observation, we assume that it generates an integer value other than the desired one with the same probability of $1 /(M-1)$.

The local sensors belonging to the same SE send signals to the fusion center in TDMA principles. Specifically, when the $k$ th local sensor of the $n$th SE assumes that its SE is at the state $H_{\tilde{m}_{n k}}$, it conveys the $\tilde{m}_{n k}$ th column of the Walsh-Hadamard matrix $\boldsymbol{W}=\left[\boldsymbol{w}_{0}, \boldsymbol{w}_{1}, \cdots, \boldsymbol{w}_{M-1}\right]$ to the fusion center within its allocated time-slot, where $\boldsymbol{W} \boldsymbol{W}^{T}=\boldsymbol{W}^{T} \boldsymbol{W}=\boldsymbol{I}_{M}$. Let $\boldsymbol{C}_{n}$ be an $\left(N_{s} \times M\right)$ matrix for DS spreading, which is used by all the $K$ local sensors of the $n$th SE. We assume that $N_{s}=M \times$ $S F$ with $S F$ being an integer denoting the spreading factor. Let $\boldsymbol{c}_{n}=\left[c_{n 0}, c_{n 1}, \cdots, c_{n\left(N_{s}-1\right)}\right]^{T}, c_{n i} \in\{+1,-1\}$, be an $N_{s}$-length spreading sequence assigned to SE $n$. Then, $\boldsymbol{C}_{n}$ is a block-diagonal matrix formed based on $\boldsymbol{c}_{n}$, where every block is an $S F$-length column vector containing $S F$ chips taken from $\boldsymbol{c}_{n}$. To illustrate, $\boldsymbol{C}_{n}$ is structured based on $\boldsymbol{c}_{n}$ as

$$
\begin{gathered}
\boldsymbol{C}_{n}=\frac{1}{\sqrt{S F}}\left[\begin{array}{cccc}
c_{n 0} & 0 & \cdots & 0 \\
\vdots & \vdots & \ddots & \vdots \\
c_{n(S F-1)} & 0 & \cdots & 0 \\
0 & c_{n S F} & \cdots & 0 \\
\vdots & \vdots & \ddots & \vdots \\
0 & c_{n(2 S F-1)} & \cdots & 0 \\
\vdots & \vdots & \ddots & \vdots \\
0 & 0 & \cdots & c_{n\left(N_{s}-S F\right)} \\
\vdots & \vdots & \ddots & \vdots \\
0 & 0 & \cdots & c_{n\left(N_{s}-1\right)}
\end{array}\right], \\
n=1,2, \ldots, N \\
2, N
\end{gathered}
$$

Explicitly, we have $\boldsymbol{C}_{n}^{T} \boldsymbol{C}=\boldsymbol{I}_{M}$. With the aid of the above settings, the discrete-time signal transmitted by the $k$ th local sensor of the $n$th SE within its allocated time-slot can be expressed as

$$
\boldsymbol{s}_{n k}=P_{n k} \boldsymbol{C}_{n} \boldsymbol{w}_{\tilde{m}_{n k}}, k=1, \ldots, K ; n=1, \ldots, N
$$

where $P_{n k}$ denotes the transmission power of the $k$ th sensor serving the $n$th SE. Note that, $\boldsymbol{s}_{n k}$ is an $N_{s}$-length vector.

Let us assume that all the local sensors have a similar distance from the fusion center and that all the sensors transmit using the same power. We assume that the $K$ sensors serving the same SE transmit their signals one-by-one serially in TDMA principles. Furthermore, synchronous transmission with respect to the $N$ SEs is assumed. Then, when assuming communications over flat Rayleigh fading channels, the discrete-time signal received by the fusion center within the $k$-th time-slot can be written as

$$
\begin{aligned}
\boldsymbol{r}_{k} & =\sum_{n=1}^{N} h_{n k} \boldsymbol{s}_{n k}+\boldsymbol{n} \\
& =\sum_{n=1}^{N} h_{n k} \boldsymbol{C}_{n} \boldsymbol{w}_{\tilde{m}_{n k}}+\boldsymbol{n}, k=1, \ldots, K
\end{aligned}
$$

where $\boldsymbol{r}_{k}$ is the $N_{s}$-length observation vector, $h_{n k}$ denotes the fading gain of the channel from the $k$-th local sensor monitoring the $n$th SE to the fusion center, $h_{n k}$ obeys the complex Gaussian distribution with zero mean and a variance of 0.5 per dimension. For convenience, in (3), the transmission power has been normalized to one. The Gaussian noise vector $\boldsymbol{n}$ in (3), is distributed with mean zero and a covariance matrix $\sigma^{2} \boldsymbol{I}_{N_{s}}$, where $\boldsymbol{I}_{N_{s}}$ is the $\left(N_{s} \times N_{s}\right)$ identity matrix and $\sigma^{2}=K / \gamma_{s}$ with $\gamma_{s}$ denoting the signal-to-noise ratio (SNR) per symbol. Let $b=\log _{2} M$ denote the number of bits per $M$-ary symbol. Then, we have $\gamma_{s}=b \gamma_{b}$, where $\gamma_{b}$ represents the SNR per bit. Note that, the factor of $K$ in $\sigma^{2}=K / \gamma_{s}$ explains that the total power received from $K$ local sensors of one SE is the same, regardless of the number of local sensors invoked. This means that less power is transmitted by each sensor, when each SE is observed by more sensors. Let us now consider the fusion rules.

\section{B. Fusion Detection Rules}

One of the important advantages of the MOM scheme is that it can be detected either coherently or noncoherently. In this 
paper, two relatively low-complexity detection rules are studied, including one coherent detection rule and one noncoherent detection rule. The coherent detection rule is operated in the MRC principles $[13,14]$, while the noncoherent detection rule is in the EGC principles $[13,14]$. Note that, more advanced detection rules may be employed in order to improve the detection reliability, but usually at the cost of increased complexity.

In order to detect the states of the $N$ SEs, the fusion center should know the spreading codes used by the local sensors. This can be done either via assigning the local sensors the corresponding spreading sequences by the fusion center itself, or via acquisition of the spreading sequences used by the local sensors. Based on either way, the fusion center can also know which SE is monitored by which local sensors. Upon carrying out the de-spreading using the $n$th SE's spreading code, we obtain

$$
\begin{aligned}
\boldsymbol{y}_{n k}= & \boldsymbol{C}_{n}^{T} \boldsymbol{r}_{k} \\
= & h_{n k} \boldsymbol{w}_{\tilde{m}_{n k}}+\underbrace{\sum_{n^{\prime}=1}^{N} h_{n^{\prime} k} \boldsymbol{C}_{n}^{T} \boldsymbol{C}_{n^{\prime}} \boldsymbol{w}_{\tilde{m}_{n^{\prime} k}}}_{\boldsymbol{I}_{n k}}+\underbrace{\boldsymbol{C}_{n}^{T} \boldsymbol{n}}_{\boldsymbol{n}_{n k}} \\
& k=1, \ldots, K ; n=1,2, \ldots, N
\end{aligned}
$$

where $\boldsymbol{y}_{n k}, \boldsymbol{I}_{n k}$ and $\boldsymbol{n}_{n k}$ are all $M$-length vectors. In (4), $h_{n k} \boldsymbol{w}_{\tilde{m}_{n^{\prime} k}}$ is the desired signal from the $k$ th sensor serving SE $n, I_{n k}$ is the interference from the sensors serving the other SEs and $\boldsymbol{n}_{n k}$ is due to noise.

When processing (4) using the Walsh-Hadamard matrix $W$, we obtain

$$
\begin{aligned}
\boldsymbol{z}_{n k}= & \boldsymbol{W}^{T} \boldsymbol{y}_{n k} \\
= & h_{n k} \boldsymbol{W}^{T} \boldsymbol{w}_{\tilde{m}_{n k}}+\boldsymbol{W}^{T}\left(\boldsymbol{I}_{n k}+\boldsymbol{n}_{n k}\right), \\
& \quad k=1, \ldots, K ; n=1,2, \ldots, N
\end{aligned}
$$

where $z_{n k}$ is an $M$-length vector, one of its entries matches to $\boldsymbol{w}_{\tilde{m}_{n k}}$ transmitted by the $k$ th sensor of SE $n$ and, hence, it contains the desired signal, interference and Gaussian noise. By contrast, all the other entries in $z_{n k}$ contain only undesired interference and Gaussian noise.

Finally, at the fusion center, the signals received from the $K$ local sensors monitoring one SE are combined to form the decision variables. Specifically, when the fusion center employs the channel knowledge $\left\{h_{n k}\right\}$ of all the channels from the local sensors to the fusion center, the MRC can be employed, which generates the decision variable vector

$$
\begin{aligned}
\boldsymbol{z}_{n}=\Re\left\{\sum_{k=1}^{K} h_{n k}^{*} \boldsymbol{z}_{n k}\right\} \\
=\Re\left\{\sum_{k=1}^{K}\left[\left|h_{n k}\right|^{2} \boldsymbol{W}^{T} \boldsymbol{w}_{\tilde{m}_{n k}}+h_{n k}^{*} \boldsymbol{W}^{T}\left(\boldsymbol{I}_{n k}+\boldsymbol{n}_{n k}\right)\right]\right\} \\
\quad n=1,2, \ldots, N
\end{aligned}
$$

The MRC rule requires the channel knowledge, which possibly demands extremely high complexity when there are many sensors. In order to attain lower complexity detection, the EGC rule may be employed, which is not dependent on the channel knowledge. With the EGC rule, the decision vector is formed as

$$
\begin{aligned}
\boldsymbol{z}_{n}= & \sum_{k=1}^{K}\left|\boldsymbol{z}_{n k}\right|^{2} \\
= & \sum_{k=1}^{K}\left|h_{n k} \boldsymbol{W}^{T} \boldsymbol{w}_{\tilde{m}_{n k}}+\boldsymbol{W}^{T}\left(\boldsymbol{I}_{n k}+\boldsymbol{n}_{n k}\right)\right|^{2} \\
& \quad n=1,2, \ldots, N
\end{aligned}
$$

when square-law based detection is considered.

Finally, based on the decision variable vector $\boldsymbol{z}_{n}, n=$ $1, \ldots, N$, the largest of its entries is selected and mapped to an integer in the range $[0, M-1]$, which represents the estimate to the state of the $n$th SE.

\section{ANALYSis OF CHARACTERISTICS}

The MOM DS-CDMA WSN proposed in this contribution employs a range of characteristics. First, it simultaneously monitors multiple SEs each with multiple parameters (states). Second, there is no interference among the $K$ sensors serving the same SE, since they communicate with the fusion center based on the TDMA principles. However, there exists interference among the sensors that serve different SEs and transmit signals simultaneously. In the proposed MOM DSCDMA WSN, this interference is mitigated with the aid of the spread-spectrum technique. Furthermore, it is well-known that the MOM is a typical on-off modulation scheme, which also has some capability to reduce interference. Third, in the MOM DS-CDMA WSN, signals transmitted from the local sensors to the fusion center are the DS spread-spectrum signals, which has the properties of low power-spectral density (PSD) of Gaussian noise alike, wideband, interference suppression, etc. Most importantly, the MOM DS-CDMA may be implemented to follow the requirements of ultra-wide bandwidth (UWB) communications. In this case, the MOM DS-CDMA WSN may be developed without requiring the license of using the frequency spectrum.

Forth, as mentioned previously, the MOM DS-CDMA WSN is suitable for using either coherent fusion rules or noncoherent fusion rules. For the MOM DS-CDMA WSNs with a low number of sensors per SE, if channel estimation is feasible, coherent fusion rules, such as MRC, may be employed in order to attain an enhanced reliability. However, when the number of sensors per SE is high, estimating the channels between sensors and fusion center may become very challenging and time and power consuming. In this case, noncoherent fusion rules will be preferred. With the noncoherent rules, although $3 \mathrm{~dB}$ of extra power is usually required for them to reach a similar detection reliability as the corresponding coherent rules, however, noncoherent fusion rules do not require overheads for channel estimation, which fundamentally saves energy. Note that, the overheads for channel estimation may become huge, when the number of sensors per SE is high. Furthermore, for those low-rate WSNs transmitting signals in burst fashion, the percentage of overheads against useful data may also be significant. For these types of WSNs, noncoherent fusion rules may also be preferred.

Fifth, as the other WSNs [1,2], in the MOM DS-CDMA WSN, the WSN's overall reliability is jointly determined by the detection reliability of local sensors and that of fusion center. If the detection reliability of the local sensors is low, then, the WSN's overall detection reliability will most probably 


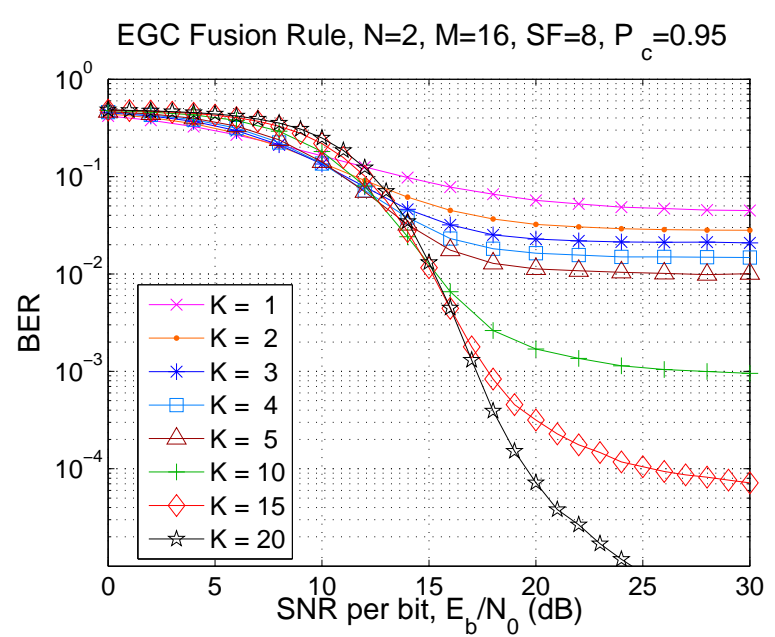

Fig. 2. EGC: BER versus average SNR per bit performance of the MOM DS-CDMA WSN, when communicating over Rayleigh fading channels.

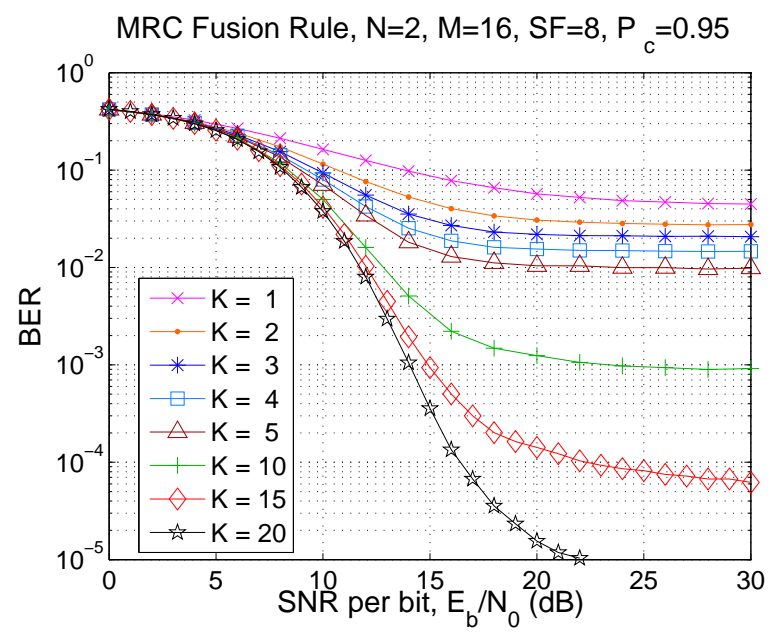

Fig. 3. MRC: BER versus average SNR per bit performance of the MOM DS-CDMA WSN, when communicating over Rayleigh fading channels.

low, regardless of the detection reliability of the fusion center. Similarly, no matter how reliably detected at the local sensors, the WSN's overall detection reliability will degrade, if the detection at the fusion center becomes unreliable. Hence, when considering the optimization of the MOM DS-CDMA WSN's overall detection performance, the fusion detection and the local sensors' detection need to be jointly optimized. Note that, the optimization issue is beyond the scope of this contribution, which, however, constitutes one of our future research topics in the context of the MOM DS-CDMA WSN.

\section{Simulation Results}

In this section, we provide a range of performance results obtained via simulations for characterizing the achievable error performance of the MOM DS-CDMA WSNs employing either EGC or MRC fusion rule. The parameters used for setting the simulations are shown associated with the figures.

Figs. 2 and 3 demonstrate the BER versus average SNR per bit performance of the MOM DS-CDMA WSN employing the EGC (Fig. 2) or MRC (Fig. 3) fusion rule, when the local sensors have a correct observation rate of $P_{c}=0.95$. First, when comparing Fig. 2 with Fig. 3, we can find that, for a given number of local sensors per SE, the error performance obtained by the MRC fusion rule is slightly better than that attained

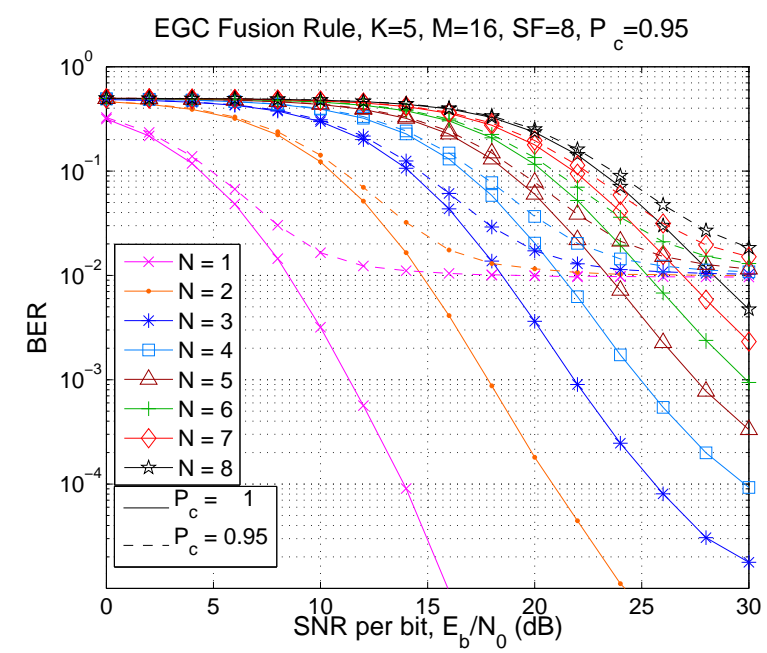

Fig. 4. EGC: BER versus average SNR per bit performance of the MOM DS-CDMA WSN, when communicating over Rayleigh fading channels.

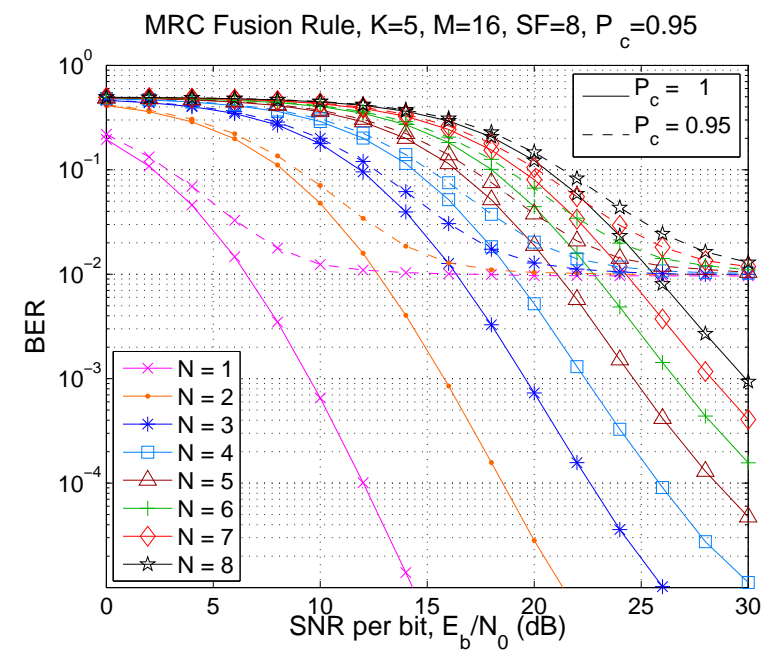

Fig. 5. MRC: BER versus average SNR per bit performance of the MOM DS-CDMA WSN, when communicating over Rayleigh fading channels.

by the EGC fusion rule. For both the fusion rules, the error performance explicitly improves as the number of local sensors per SE increases, due to the increased spatial diversity. When the number of local sensors is not high, error-floors appear. The reason behind is as follows. First, at a given time, there are two sensors belonging to the two SEs, which send their signals simultaneously and hence interfere with each other. Second, the local sensors make error observation with an error rate $P_{e}=1-0.95=0.05$, which results in that the fusion center makes erroneous decisions, even when the channels from the local sensors to the fusion center are ideally reliable.

Figs. 4 and 5 show the BER versus average SNR per bit performance of the MOM DS-CDMA WSNs employing the EGC (Fig. 4) or MRC (Fig. 5) fusion rule, when the WSNs monitor different number of SEs simultaneously. Both the local sensors without observation errors $\left(P_{c}=1\right)$ and that with a correct observation probability of $P_{c}=0.95$ are addressed. Explicitly, the reliability of the fusion detection degrades as the number of SEs monitored simultaneously by the fusion center increases. When $P_{c}=1$, the BER of fusion detection may go very low, as the SNR increases. However, when $P_{c}=0.95$, the BER of fusion detection is constrained by the error rate of local sensors' detection. The BER cannot be reduced further by 


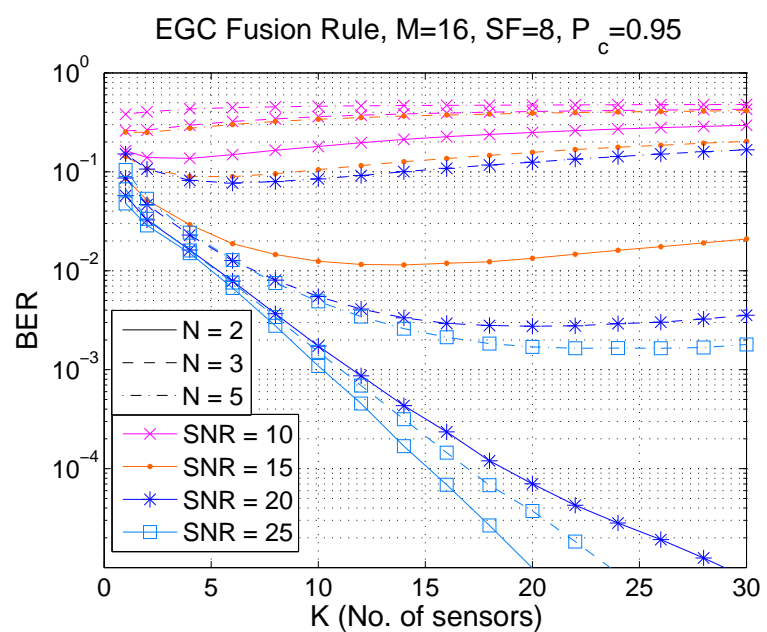

Fig. 6. EGC: BER versus average SNR per bit performance of the MOM DS-CDMA WSN, when communicating over Rayleigh fading channels.

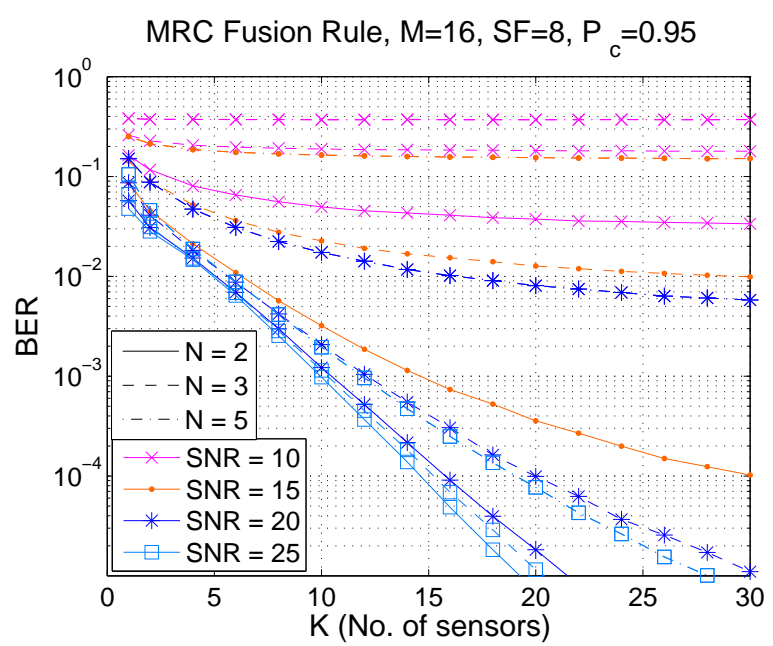

Fig. 7. MRC: BER versus average SNR per bit performance of the MOM DS-CDMA WSN, when communicating over Rayleigh fading channels.

increasing the SNR, after reaching a certain value.

Finally, in Figs. 6 and 7, we illustrate the impact of the number of local sensors per SE on the detection reliability of the fusion detection, when the EGC (Fig. 6) or MRC (Fig. 7) fusion rule is employed. As seen in Figs. 6 and 7, different numbers of SEs and different values of SNR are considered. From the results of these figures, we can see that, when the channel SNR is low, which makes the transmissions from the local sensors to the fusion center highly unreliable, then, the WSNs are unreliable, no matter how many local sensors per SE are employed. By contrast, if the channel SNR is sufficiently high, then, reliable fusion detection is available by deploying a sufficient number of local sensors for monitoring every SE. Again, the results of these figures show that, when the values of SNR and $K$ are fix, the fusion detection becomes less reliable, as the number of SEs monitored increases.

\section{CONCLUSION}

In summary, a WSN framework has been proposed, which monitors multiple $M$-ary SEs whose states are conveyed via local sensors to the fusion center in the principles of TDMA and DS-CDMA. The DS-CDMA has been introduced to allow several local sensors to transmit signals simultaneously to the fusion center. The MOM scheme is employed in favor of either coherent or noncoherent detection. The error performance of the MOM DS-CDMA WSN has been investigated and the effects of different aspects have been observed. Our studies show that the MOM DS-CDMA WSN is capable of achieving a promising detection performance for reasonable channel SNR. However, the detection performance of the MOM DS-CDMA WSN is jointly determined by the local sensors' reliability and the fusion center's reliability. Hence, when the total power of the WSN is given, the local sensors' detection strategy and the fusion center's detection strategy need to be jointly optimized. This constitutes one of our future research topics in the context of the MOM DS-CDMA WSNs.

\section{REFERENCES}

[1] A. Swami, Q. Zhao, Y.-W. Hong, and L. Tong, Wireless Sensor Networks: Signal Processing and Communications Perspectives. Chichester, England: John Wiley \& Sons, 2007.

[2] R. Viswanathan and P. K. Varshney, "Distributed detection with multiple sensors: Part i-Fundamentals," Proc. of the IEEE, vol. 85, no. 1, pp. 54 63, Jan. 1997.

[3] B. Chen, R. Jiang, T. Kasetkasem, and P. K. Varshney, "Channel aware decision in wireless sensor networks," IEEE Trans. on Signal Proc., vol. 52, no. 12 , pp. $3454-3458$, Dec. 2004.

[4] R. Niu, B. Chen, and P. K. Varshney, "Fusion of decisions transmitted over Rayleigh fading channels in wireless sensor networks," IEEE Trans. on Signal Proc., vol. 54, no. 3, pp. 1018 - 1027, Mar. 2006.

[5] B. Chen, L. Tong, and P. K. Varshney, "Channel-aware distributed detection in wireless sensor networks," IEEE Signal Proc. Mag., no. 3, pp. 16 -26, July 2006.

[6] Y. Lin, B. Chen, and P. K. Varshney, "Decision fusion rules in multi-hop wireless sensor networks," IEEE Trans. on Aero. and Elec. Sys., vol. 41, no. 2, pp. 475 - 487, Apr. 2005

[7] T.-Y. Wang, Y. Han, B. Chen, and P. K. Vaeshney, "A combined decision fusion and channel coding scheme for distributed fault-tolerant classification in wireless sensor networks," IEEE Trans. on Wireless Comm., vol. 5, no. 7 , pp. 1695 - 1705 , July 2006.

[8] R. Jiang and B. Chen, "Fusion of censored decisions in wireless sensor networks," IEEE Trans. on Wireless Comm., vol. 4, no. 6, pp. 2668 -2673, Nov. 2005.

[9] S. A. Aldosari and J. M. F. Moura, "Detection in sensor networks: the saddlepoint approximation," IEEE Trans. on Signal Proc., vol. 55, no. 1 , pp. 327 - 340, Jan. 2007.

[10] V. Veeravalli and J.-F. Chamberland, "Detection in sensor networks," in Wireless Sensor Networks: Signal Processing and Communications Perspectives, Y.-W. H. A. Swami, Q. Zhao and L. Tong, Eds. John Wiley \& Sons, 2007, pp. $119-148$

[11] W. Li and H. Dai, "Distributed detection in wireless sensor networks using a multiple access channel," IEEE Trans. on Signal Processing, vol. 55 , no. 3, pp. 822 - 833, March 2007

[12] J. G. Proakis, Digital Communications, 3rd ed. McGraw Hill, 1995.

[13] L.-L. Yang, Multicarrier Communications. Chichester, United Kingdom: John Wiley, 2009.

[14] M. K. Simon and M.-S. Alouini, Digital Communication over Fading Channels: A Unified Approach to Performance Analysis. New York: John Wiley \& Sons, 2000. 